\title{
Engineering Service Oriented Applications: From StPowla Processes to SRML Models ${ }^{\star}$
}

\author{
Laura Bocchi $^{1}$, Stephen Gorton ${ }^{1,2}$, and Stephan Reiff-Marganiec ${ }^{1}$ \\ ${ }^{1}$ Department of Computer Science, University of Leicester \\ University Road, Leicester LE1 7RH, UK \\ \{bocchi, smg24, srm13\}@mcs. le.ac.uk \\ ${ }^{2}$ ATX Technologies Ltd, MLS Business Centres, \\ 34-36 High Holborn, London WC1V 6AE, UK
}

\begin{abstract}
Service Oriented Computing is a paradigm for developing software systems as the composition of a number of services. Services are loosely coupled entities, can be dynamically published, discovered and invoked over a network. The engineering of such systems presents novel challenges, mostly due to the dynamicity and distributed nature of service-based applications. In this paper, we focus on the modelling of service orchestrations. We discuss the relationship between two languages developed under the SENSORIA project: SRML as a high level modelling language for Service Oriented Architectures, and STPOWLA as a process-oriented orchestration approach that separates core business processes from system variability at the end-user's level, where the focus is towards achieving business goals. We also extend the current status of STPOWLA to include workflow reconfigurations. A fundamental challenge of software engineering is to correctly align business goals with IT strategy, and as such we present an encoding of STPOWLA to SRML. This provides a formal framework for STPOWLA and also a separated view of policies representing system variability that is not present in SRML.
\end{abstract}

\section{Introduction}

Service Oriented Computing (SOC) is a paradigm for developing software systems as the composition of a number of services, that are loosely coupled entities that can be dynamically published, discovered and invoked over a network. A service is an abstract resource whose invocation triggers a possibly interactive activity (i.e. a session) and that provides some functionality meaningful from the perspective of the business logic [8]. A Service Oriented Architecture (SOA) allows services with heterogeneous implementations to interact relying on the same middleware infrastructure. Web Services and the Grid are the most popular implementations of SOA. Exposing software in this way means that applications may outsource some functionalities and be dynamically assembled, leading to massively distributed, interoperable and evolvable systems.

The engineering of service-oriented systems presents novel challenges, mostly due to this dynamicity [20]. In this paper we focus on the modelling of orchestrations. An orchestration is the description of the executable pattern of service invocations/interactions to follow in order to achieve a business goal.

\footnotetext{
^ This work has been partially sponsored by the project SENSORIA, IST-2005-016004.
} 
We discuss the relationship between two modelling languages for service oriented systems developed in the context of SENSORIA, an IST-FET Integrated Project on Software Engineering for Service-Oriented Overlay Computers: the SENSORIA Reference Modelling Language (SRML) [51] and STPOWLA: the Service-Targeted, PolicyOriented WorkfLow Approach [6]. We also discuss the advantages of their combined usage.

SRML is a high-level modelling language for SOAs whose goal is "to provide a set of primitives that is expressive enough to model applications in the service-oriented paradigm and simple enough to be formalized" [5]. SRML aims at representing, in a technology agnostic way, the various foundational aspects of SOC (e.g. service composition, dynamic reconfiguration, service level agreement, etc.) within one integrated formal framework.

STPOWLA is an approach to process modelling for service-oriented systems. It has three ingredients: workflows to express core processes, services to perform activities and policies to express variability. Workflows are expressed using a graphical notation, such as in [7]. Policies can make short-lived changes to a workflow instance, i.e. they last for the duration of the workflow instance and usually will be made during the execution of the instance, rather than applied to the overall workflow model.

So far, STPOWLA has been limited to non-functional changes to a workflow. In this paper, we extend the concept of workflow change to include reconfigurations: short lived structural changes to a workflow instance. We substantiate this extension by defining a further encoding of these advanced control flow aspects into SRML.

The encoding of STPOWLA into SRML provides a formal framework to STPOWLA. Business processes modelled in STPOWLA can be represented as SRML models and either analyzed alone or as part of more complex modules, where they are composed with other SRML models with heterogeneous implementations (e.g. SRML models extracted from existing BPEL processes [3]).

A second reason for the encoding is providing a higher layer to the modelling of orchestrations in SRML that includes a process-based approach to the definition of a workflow schedule, a separated view of policies, that had not been yet considered in SRML, and the inter-relation between workflow and policies.

In this paper, we give an overview of the STPOWLA approach, including the extension for workflow reconfigurations in section 2. We describe the main concepts of SRML, with respect to STPOWLA in section 3 . We then provide an encoding of basic workflow control flow constructs in section 4 , and proceed to describe STPOWLA reconfigurations as advanced control flow encodings in section 5. We describe related work and thus our position relative to these efforts in section 6 before discussing and concluding in sections 7 and 8 .

\section{Specifying and Reconfiguring StPowla Workflows}

In this section, we give a brief introduction to the main concepts of STPOWLA. In addition, we present the concept of workflow reconfiguration, which is an extension to the current state of STPOWLA. 


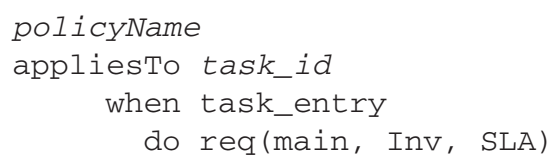

Fig. 1. A STPOWLA task's default policy. The semantics of the req function are essentially to execute the processing of the task, as specified with functional requirements described in the main argument, in accordance with invocation parameters in the second argument and keeping to default SLA constraints in the third argument.

\subsection{Overview}

STPOWLA has three ingredients: workflows, SOA and policies. Workflows specify core business processes, in which all task requirements are satisfied by services. Each workflow task has a default policy as in Fig. 1 .

We describe a workflow, according to [7], with the following grammar to show how complex processes can be composed:

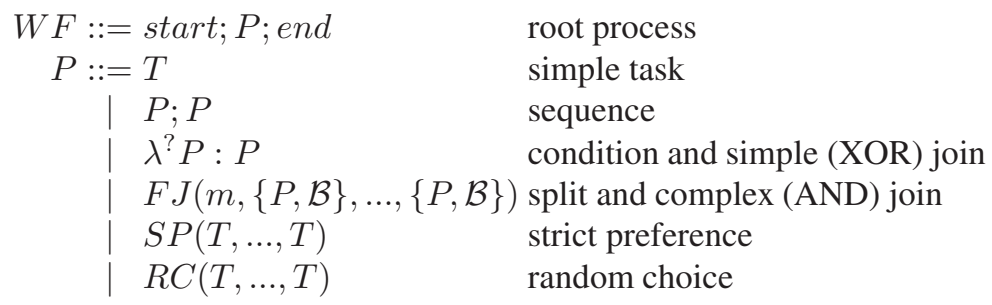

We describe the semantics of each construct with a description of the relevant SRML transition in section 4.

Policies are either Event-Condition-Action (ECA) rules (in which case they require a trigger), or goals (essentially ECAs without triggers). The purpose of policies is to express system variability. Policies are written in APPEL [14], a policy description language with formal semantics via a mapping to $\triangle D S T L[10]$. They are written by the end (business) user and are added and removed at any time to the workflow. In addition to default policies, other policies can be added to the workflow to express system variability in terms of refinement and reconfiguration. The former type express constraints over runtime execution and service selection, but is out of the scope of this paper.

We have mentioned in an earlier paper [6] that the choice of workflow notation in STPOWLA is of small significance. What is of interest is the identification of a common set of triggers for ECA policies. We have identified the following as valid triggers:

- Workflow entry/success/failure/abort;

- Task entry/success/failure/abort;

- Service entry/success/failure.

Note that in STPOWLA, we view services as a black box, i.e. we cannot intervene in their processing between invocation and (possible) response. 
Table 1. Policy reconfiguration functions

\begin{tabular}{|c|c|}
\hline Function Syntax & Informal Description \\
\hline fail() & $\begin{array}{l}\text { Declare the current task to have failed, i.e. discard further task } \\
\text { processing and generate the task_failure event. }\end{array}$ \\
\hline abort ( ) & $\begin{array}{l}\text { Abort the current task and progress to the next task, generating } \\
\text { the task_abort event. }\end{array}$ \\
\hline block $(s, p)$ & Wait until predicate $\mathrm{p}$ is true before commencing scope $\mathrm{s}$. \\
\hline insert $(x, y, z)$ & $\begin{array}{l}\text { Insert task or scope } \mathrm{y} \text { into the current workflow instance after } \\
\text { task } \mathrm{x} \text { if } \mathrm{z} \text { is true, or in parallel with } \mathrm{x} \text { if } \mathrm{z} \text { is false. }\end{array}$ \\
\hline delete $(\mathrm{x})$ & Delete scope $\mathrm{x}$ from the current workflow instance. \\
\hline
\end{tabular}

\subsection{Reconfiguring Workflows with Policies}

A workflow reconfiguration is the structural change of a workflow instance. In STPOWLA, a policy can express a reconfiguration rule based on a number of available functions, as described in Table 1. These changes are short-lived, i.e. they only affect the workflow instance and not the overall workflow model.

As an example, consider a supplier whose business process is to receive an order from a registered customer, and then to process that order (which includes collecting, packing and shipping the items, plus invoicing the client). There are no extra constraints on each task, therefore the default task policies are effectively "empty".

Now consider that under certain conditions (e.g. financial pressure), a financial guarantee is required from all customers whose order is above a certain amount. We may have the following policy:

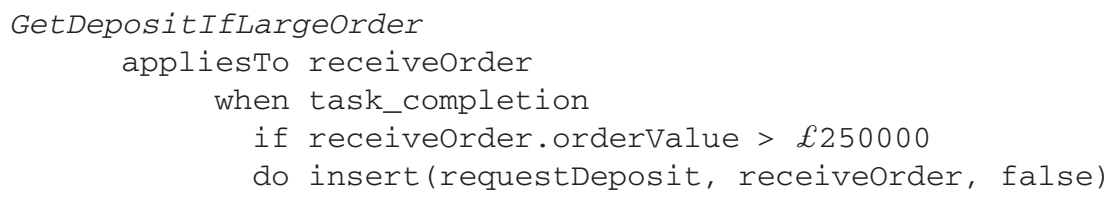

Intuitively, this policy (named GetDepositIfLargeOrder) applies to the receiveOrder task. It says that when the task completes successfully and the attribute orderValue (bound to that task) is above $£ 250000$, then there should be an action. The action in this case is the insertion of a task requestDeposit into the workflow instance after (not in parallel to) the receiveOrder task. The workflow instance thus undergoes the transformation as shown in Fig. 2 .

\section{Encoding of StPowla to SRML - Foundational Concepts}

In SRML, composite services are modelled through modules. A module declares one or more components, that are tightly bound and defined at design time, a number of requires-interfaces that specify services, that need to be provided by external parties, and (at most) one provides-interface that describes the service that is offered by the 


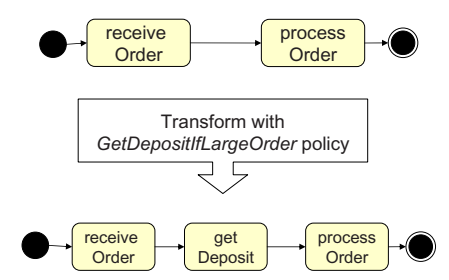

Fig. 2. A simple reconfiguration example where a core business process is transformed via the insertion of the getDeposit task after the receiveOrder task. The transformation rule comes from a policy.

module. A number of wires establish interaction protocols among the components and between the components and the external interfaces.

Components, external interfaces and wires are specified in terms of Business Roles, Business Protocols and Interaction Protocols, respectively. The specifications define the type of the nodes.

In this paper we provide an encoding to derive, from a business process specified in STPOWLA, an SRML component that we call BP, of type businessProcess and a second component $P I$, of type policyInter face that is connected to $B P$ and represents the interface through which it is possible to trigger policies that modify the control flow. $P I$ supports the set of interactions used to trigger a workflow modification in the component BP. Fig. 3 illustrates the structure of the SRML module representing the workflow and policies in the procurement example described earlier in this section.

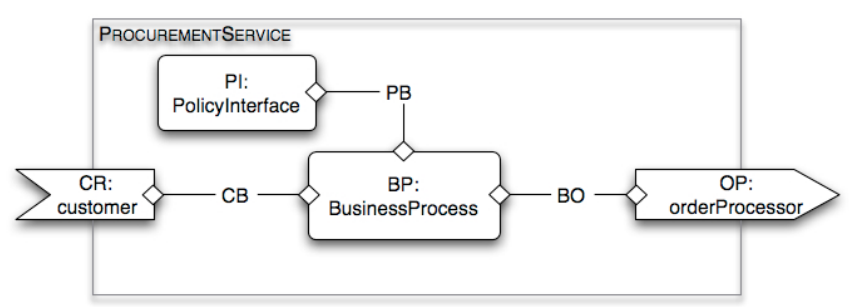

Fig. 3. The structure of a SRML module for the procurement service example

Components are instances of Business Roles specified in terms of (1) the set of supported interactions, (2) the way in which the interactions are orchestrated. We provide in the rest of this section an overview of Business Roles. The overview will not involve the other types of specification as they are not concerned in the encoding.

Business Roles: the Interactions. SRML supports asynchronous two-way conversational interactions: $\mathbf{s} \& \mathbf{r}$ denotes interactions that are initiated by the co-party, which expects a reply, r\&s denotes interactions that are initiated by the party, which expects a reply from its co-party. SRML supports also asynchronous one-way and synchronous interactions that are not discussed here as they are not involved in the encoding. 
4. The sends sentence describes the events that are sent and the values taken by their parameters. In the example we invoke the samplePolicy reply event to notify the correct management of the policy.

\section{Basic Control Flow Encoding}

In this section we present an encoding from the control constructs of STPOWLA to SRML orchestrations. Our focus is on the control constructs and we abstract from the interactions of the service and from the semantics of the simple activities of the workflow tasks.

STPOWLA represents a business process as the composition of a number of tasks, either simple (e.g. interactions with services) or complex (e.g. coordinating other tasks by executing them in sequence, parallel, etc.). In SRML we associate an identifier, of type task $I d$, to any task. We denote with $T$ the set of all the task indexes in the workflow schedule.

For every task identifier $x$ we define the following local variables, used to handle the control flow and coordinate the execution of the tasks:

- start $[x]$ is a boolean variable that, when true, triggers the execution of $x$;

- done $[x]$ is a boolean variable that signals the successful termination of $x$ and triggers the continuation of the workflow schedule;

- $f a i l[x]$ is a boolean variable that signals the termination with failure of $x$ and triggers the failure handler.

In general, the next activity in the control flow is executed when the previous one terminates successfully. In case of task failure the flow blocks (i.e. the next task is waiting for a signal of successful termination from the previous task) and the failure signal is collected by a failure handler that possibly involves a number of policies. According to the failure handler, the execution of the process can be terminated, resumed, altered, etc. We leave the specification of the failure handling mechanisms as a future work. Anyway, the constructs of strict preference and random choice, that try a number of alternative tasks until one terminates with success, handle the failure signal directly within the workflow.

We will introduce in section 5 a set of transitions, as a part of the orchestration of $B P$ that model the policy handler. The policy handler has the responsibility to enact the modifications of the control flow induced by the policies triggered by $P I$. The policy handler blocks the normal flow by setting the variable policy $[x]=\operatorname{true}$, where $x$ is the identifier of the first task involved in the modification. The variable policy $[x]$ is a guard to the execution of $x$. We will describe the policy handler more later in this paper, but now it is important to know that when a policy function has to be executed on a task, the task has to be blocked. It is responsibility of the policy handler to reset the flow of execution.

Some policies can be applied only on running processes (e.g. abort) and some others only on tasks that have not started yet (e.g. delete). A local variable state $[x]$ identifies the state of the execution of $x$ by taking the values toStart (i.e. the execution of the task has not started yet), running (i.e. the task is in execution) and exited (i.e. $x$ has 
terminated). The state variable state $[x]$ will be used to ensure that policies act on a task in the correct state of execution.

We consider the simple tasks as black boxes: we are not interested in the type of activity that they perform but only on the fact that a task, for example task $x$, is activated by start $[x]$, signals its termination along either done $[x]$ or failed $[x]$ and notifies its state along state $[x]$.

The execution of the workflow is started by a special transition root that sets $\operatorname{start}[x]=$ true where $x$ is the first task in the workflow schedule. The local variables are initialized as follows: $\forall i \in T \backslash \operatorname{root}$, start $[i]=$ false $\wedge$ start $[$ root $]=$ true, $\forall i \in T$, done $[i]=$ failed $[i]=$ policy $[i]=$ false and $\forall i \in T$, state $[i]=$ toStart.

It follows the encoding of the workflow template start; $P$; end where $P$ is associated to the task identifier $x$ :

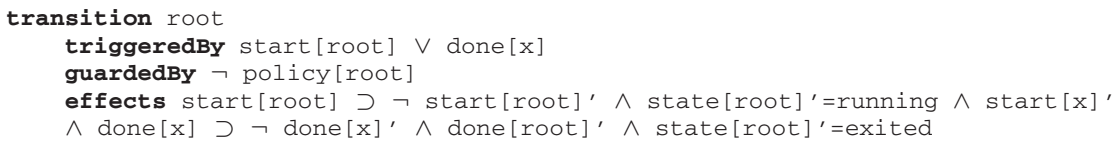

Sequence. The sequence operator $P_{1} ; P_{2}$, illustrated in Fig. 4 first executes $P_{1}$ and, after the successful termination of $P_{1}$, executes $P_{2}$. We remark that failures are not handled in this document and will be addressed in the future.

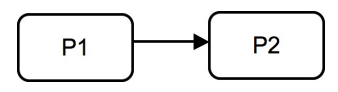

Fig. 4. The sequence control construct in STPOWLA

The encoding of the sequence construct in SRML is as follows. The sequence activity triggers the execution of the first task, with task identifier $p 1$, then collects the termination signal from $p 1$ and triggers the execution of the second subprocess, with task identifier $p 2$. The sequence is encoded in the following SRML transition, with task identifier $x$ :

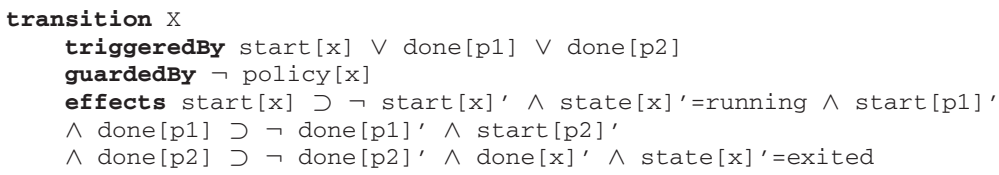

Condition and Simple Join (XOR). The condition and simple join construct $\lambda^{?} P_{1}$ : $P_{2}$, illustrated in Fig. 5(a), consists of the combination of the flow junction, that diverts the control flow down one of two branches $P_{1}$ and $P_{2}$, represented by the task identifiers $p 1$ and $p 2$, respectively, according to a condition $\lambda$, and the flow merge of a number of flows where synchronization is not an issue. 


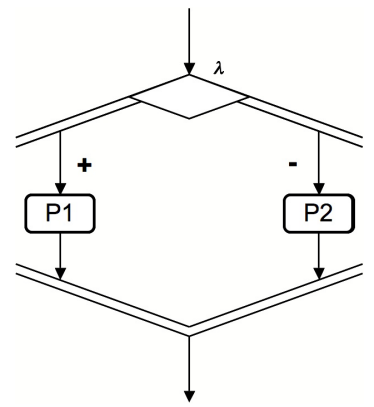

(a) Condition/simple join

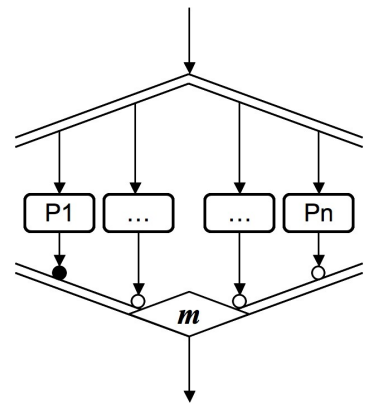

(b) Split/complex join

Fig. 5. Multiple branches constructs in STPOWLA

The condition and simple join are encoded into the following SRML transition:

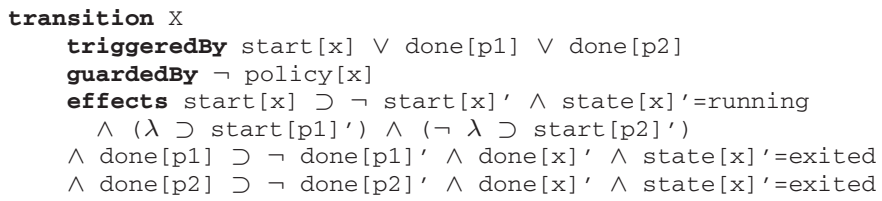

Split and Complex Join (AND). The split and complex join construct $F J\left(m,\left\{P_{1}\right.\right.$, $\left.\left.\mathcal{B}_{1}\right\}, \ldots,\left\{P_{n}, \mathcal{B}_{n}\right\}\right)$ consists of the combination of the flow split, that splits the control flow over many branches, and the conditional merge, that synchronizes two or more flows into one. The value of $m$, that is statically determined, represents the minimum number of branches that have to be synchronized. Furthermore, any branch is associated to a boolean $\mathcal{B}_{i}$ that determines whether the $i-t h$ branch is mandatory in the synchronization. The graphical notation of the construct is illustrated in Fig. 5(b)

The encoding is as follows: Let $S$ be the set, with cardinality $n$, of the task indexes associated to the branches of the split/join. Let the identifiers for the subtasks of $x$ to range over $p 1, \ldots, p n$. Let $N$ be the set of indexes of the necessary tasks and $m \in \mathbb{N}$ be the minimum number of branches that have to be synchronized. We assume that $m \leq$ $|N|$. The complex join is encoded in the following SRML transition, where Kcomb is the set of $(m-|N|)-$ subsets of $S \backslash N$ :

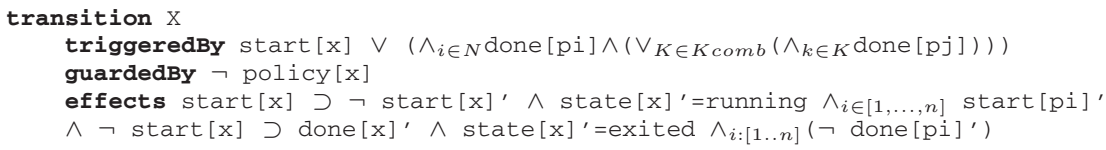

The transition is executed: (1) when the task $x$ is triggered or (2) in case of successful termination of all the necessary subtasks (i.e. $\wedge_{i \in N}$ done $[p i]$ ) and of a number of tasks greater or equal to $m$ (i.e. $\vee_{K \in K \operatorname{comb}}\left(\wedge_{k \in K}\right.$ done $\left.\left.[p j]\right)\right)$.

Strict Preference. The strict preference $S P\left(P_{1}, \ldots, P_{n}\right)$, illustrated in Fig. 6(a), attempts the tasks $P_{1}, \ldots, P_{n}$ one by one, in a specific order, until one completes successfully. In this case, with no loss of generality we consider the tasks ordered by increasing index numbers. 


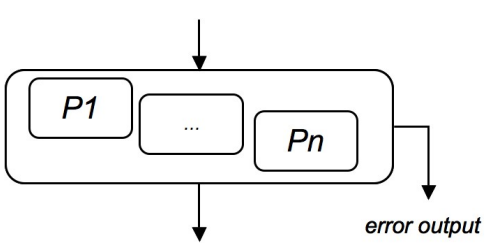

(a) Strict preference

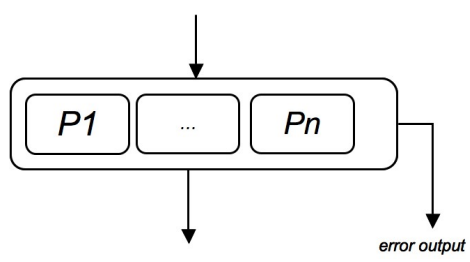

(b) Random choice

Fig. 6. Other constructs in STPOWLA

The strict preference is encoded in the following SRML transition:

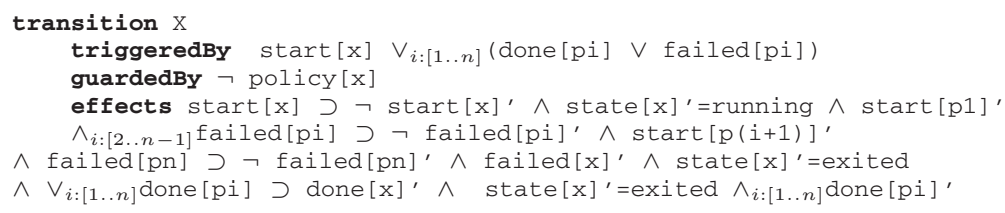

Random Choice. The random choice $R C\left(P_{1}, \ldots, P_{n}\right)$, illustrated in Fig. 6(b), attempts the tasks $P_{1}, \ldots, P_{n}$ simultaneously and completes when one completes successfully.

The random choice is encoded in the following SRML transition:

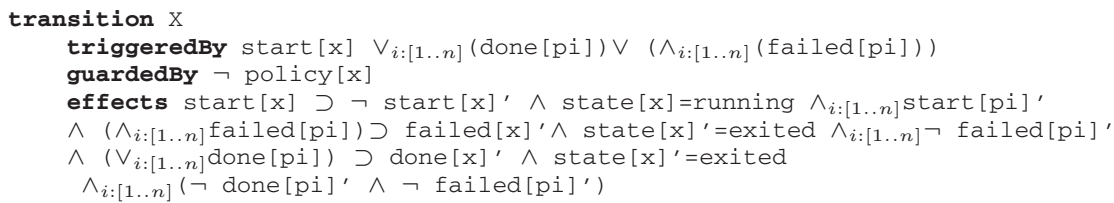

\section{Advanced Control Flow Encoding}

One of the aims of this paper is to illustrate how policies can influence the control flow and how this can be modelled in SRML. In this section we discuss the encoding of policies, as described in STPOWLA, into SRML orchestrations. Each interaction is handled, in the orchestration of $B P$, by one or more transitions that model the policy handler. We will see such transitions in detail when discussing individual interactions, in the rest of this section.

A policy related to a task can have effect (1) on the state prior to the task execution (i.e. delete, block and insert) or (2) during the execution of a task (i.e. fail and abort). The state of a task is notified along the variable state $[y]$. The policy handler must check that the task is in the correct state according to the specific policy that has to be enacted. The policy handler prevents the execution of either (1) the task or (2) the rest of the task by using the variable policy $[x]$ : the condition $\neg$ policy $[x]$ guards the transition(s) corresponding to the execution of task. Notice that with most of the control constructs it is not possible to trigger policies of this second type on atomic tasks whose state changed directly from toStart to done. 
Delete Task. The deletion of task (i.e. delete(x) in STPOWLA) skips the execution of $x$. The policy manager prevents the execution of $x$ by signaling a policy exception (i.e. policy $[x]=$ true). When the signal for the execution of $x$ is received, the policy handler signals the successful termination of $x$. The condition P_delete $[i] \delta$ ? is true when the event delete[i]\&? occurred in the past.

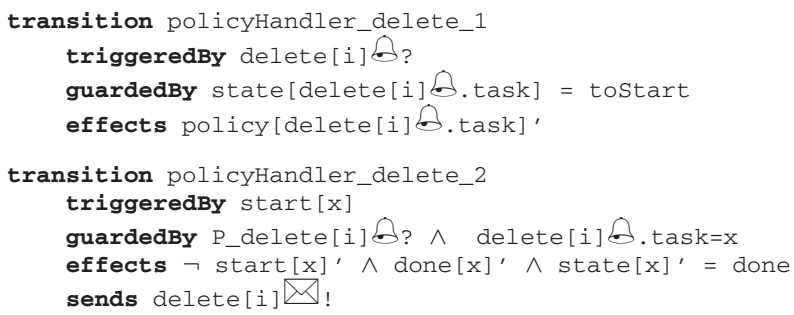

Block Task. The function $\operatorname{block}(\mathrm{x}, \mathrm{p})$ in STPOWLA blocks a task $x$ until $p$ is true. In SRML the policy handler prevents $x$ from executing (i.e. policy [x] becomes true) temporarily until $p$ is true. The policy handler notifies the enactment of the policy to the environment and after that the task has been unblocked.

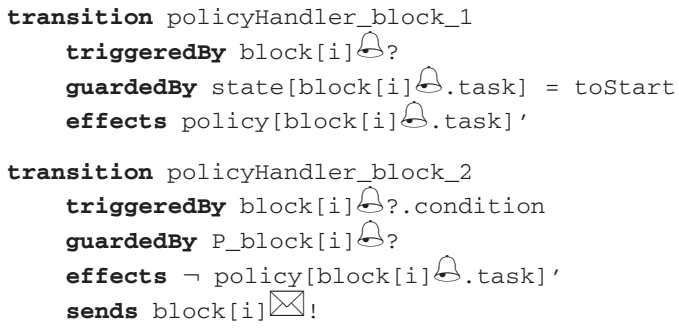

Insert Task. The insertion of a task, represented by the function insert $(\mathrm{x}, \mathrm{y}, \mathrm{z})$ in STPOWLA, inserts the task $y$ in sequence or in parallel with respect to $x$ depending on the value of the boolean variable $z$. In SRML the insertion is triggered by the interaction insert $[i] \bigotimes$ ? with parameter insert $[i] \bigotimes$.task representing the task $x$, insert $[i] \bigotimes$.insertedTask representing the task $y$ and insert $[i] \bigotimes$.condition representing the condition $z$. We assume that the set of tasks available for insertion is determined a priori, in this way we assume that the SRML encoding has a set of transitions for each possible task, including the task to possibly insert, that is executed by setting start $[y]$ to true. We introduce in this way a limitation on the number of task types that we can insert and on the fact that a task can be inserted only once (we will manage multiple insertions in the future, when we will encode looping constructs) but we do not provide any limitation on the position of the insertion.

We rely on a function next $:$ taskId $\rightarrow$ taskId that returns, given a task, the next task to execute in the workflow. Such a function can be defined by induction on the syntax of STPOWLA defined in section 2.1

The transition policyHandler_insert_1 prevents the execution of the task on which the policy applies (i.e. insert $[i] \varangle$.task) and of the successive one. The transition policyHandler_insert_2 starts the execution of the task on which the policy applies (in parallel with the inserted task if insert $[i] \triangle$.condition $=$ true). The 
transitions policyHandler_insert_sequence and policyHandler_insert_parallel coordinate the execution of the tasks (the one on which the policy applies and the inserted one) in sequence or in parallel, according to the condition.

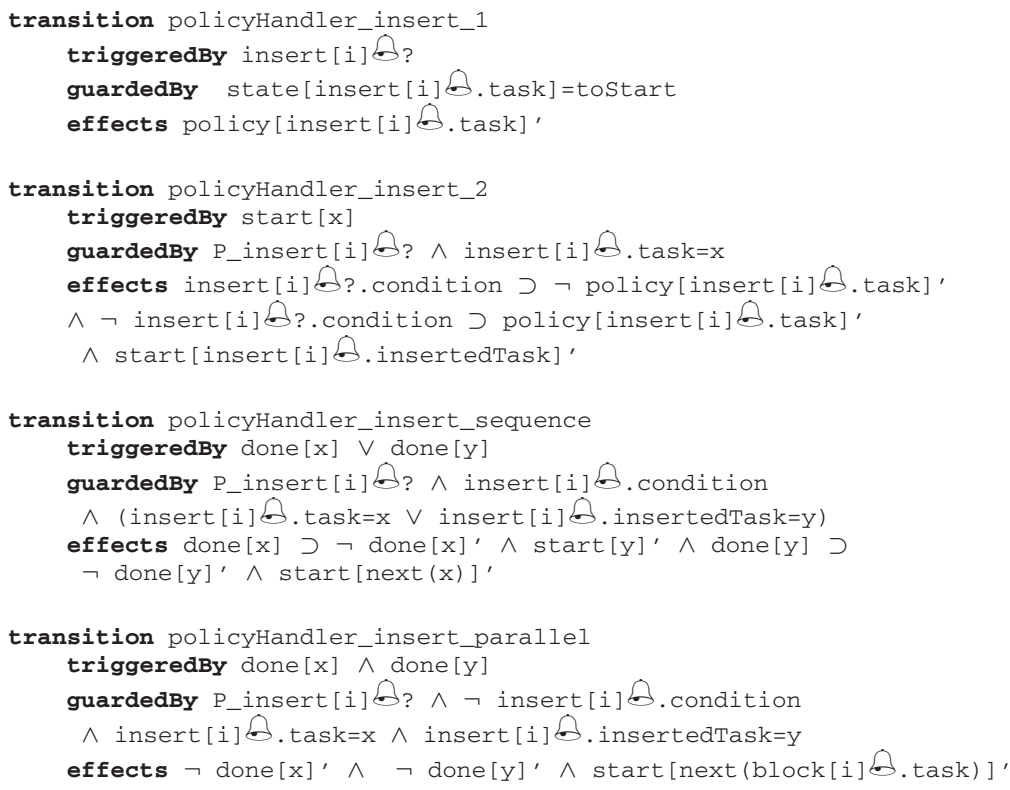

Fail Task. The failure of a task must occur during the execution of the task (it has no effect otherwise). The failure can be triggered autonomously, within the task or induced externally by the execution of the policy fail. We consider here the second case.

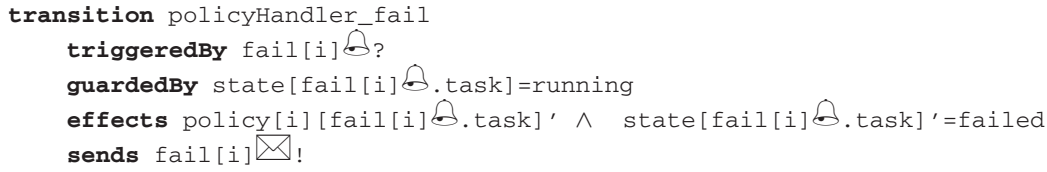

Abort Task. The abortion of a task is similar to a deletion, but it involves a running task. Aborting a task that has neither started or has already completed has no effect.

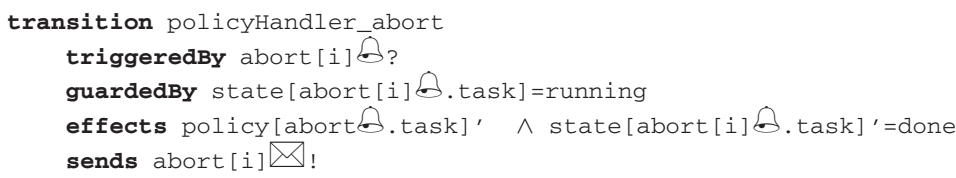

\subsection{An Example: The Reconfiguration of the Procurement Scenario}

The orchestration of the Business Role businessProtocol would consist of the sequence of the tasks request order (i.e. task ro) and process order (i.e. task $p o$ ). 


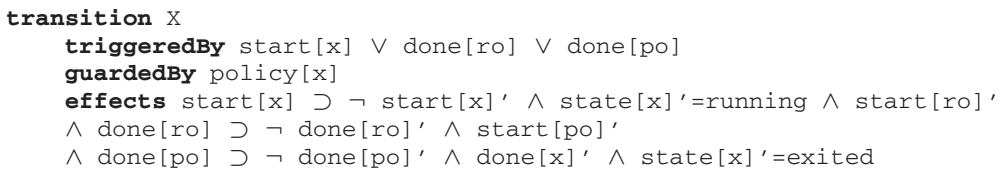

In the case of a receive event of type insert $[i] \bigotimes$ ?, triggered by the component $P I$, with parameter task equal to po, parameter insertedTask equal to gbd (i.e. get deposit), and the parameter condition equal to true. The policy handler would: (1) block the execution of $r o$ (preventing in this way ro to trigger its continuation $p o=n e x t(r o)$ ) by setting policy $[$ ro $]=\operatorname{policy}[\mathrm{po}]=$ true, (2) wait for the condition $\operatorname{start}[$ ro $]=$ true that is triggered by transition $X$, (3) since the parameter condition is true, the policy handler would unblock ro, (4) the transition policyHandler_insert_sequence would handle the execution of $g d$ after $r o$ and, finally, trigger po by setting $\operatorname{start}[p o]=$ true.

\section{Related Work}

SRML is inspired by the Service Component Architecture (SCA) [9]. SCA is a set of specifications, proposed by an industrial consortium, that describe a middlewareindependent model for building over SOAs.

Similarly to SCA, SRML provides primitives for modelling, in a technology agnostic way, business processes as assemblies of (1) tightly coupled components that may be implemented using different technologies (including wrapped-up legacy systems, BPEL, Java, etc.) and (2) loosely coupled, dynamically discovered services.

Differently from SRML, SCA is not a modelling language but a framework for modelling the structure of a service-oriented software artifact and for its deployment. SCA abstracts from the business logic provided by components in the sense that it does not provide a means to model the behavioural aspects of services. SRML is, instead, a modelling language that relies on a mathematical framework and that provides the primitives to specify such behavioural aspects.

Process modelling at a business level is generally achieved using graphical languages such as BPMN [19] or UML Activity Diagrams. However, they do not cater for all workflow patterns [17], as described in [21] and [15], respectively. YAWL [16] caters for all workflow patterns and has a graphical syntax with formal semantics, based on petri nets, so it is a good candidate for the process notation. As we have previously mentioned, the syntax is not significant, but rather the places where a policy can interact is. We have used the language of [7] for its simplicity, expressive power and our familiarity with it. At a lower business level, languages such as BPEL or WS-CDL are capable of expressing business processes, but with a code-based approach that is not high level enough for the end user.

Policies have generally been used as an administration technique in system management (e.g. access control [11] or Internet Telephony [13]). In addition, a methodology has been proposed to extract workflows from business policies [18]. However, we are not aware of policies being used as a variability factor in service-targeted processes. 
Dynamic processes that are based on the end-user's needs are more difficult to find. The closest we know of are AgentWork [12], which is dynamic only because of the choice of rules to follow under failure, and Worklets [2], the YAWL module for dynamic processes. Worklets though are based on a set of processing rules that are predefined, and of which one is selected.

\section{Discussion}

The benefits of this work are twofold. The mapping from STPOWLA to SRML creates a formal framework for the former (which previously only benefitted from a formal semantics for the APPEL policy language). Since applications are often designed based on the business process, STPOWLA is the ideal vehicle for this step. Transformation to SRML modules allows for analysis of the modules, either on their own or as part of more complex modules. Looking at the encoding bottom-up, STPOWLA adds a higher level of modelling to SRML modules in the form of a process-oriented workflow schedule, with system variability separated from the core business concerns.

To exemplify the benefits, we describe an application scenario from an industrial case study, provided by a partner in the SENSORIA PROJECT. The scenario describes the interaction between a VoIP telephony provider and their internal service network, based on a pre-delivery state.

The trigger of the workflow is the supplier receiving an order from a customer. Under normal operating circumstances, this proceeds to a testing phase, where the customer can choose to accept or decline the test results. If they accept the results, then the supplier will proceed to identifying an offer proposal. In order to do this, legal assistance is required from a legal service. This assistance is then received and embedded into a Service Level Agreement between the customer and the supplier. The contract is created and the workflow is then complete.

There are two policies that affect how the workflow is executed. Firstly, if the order is sufficiently small and the customer's order is of sufficiently small value, then the initial testing phase should be bypassed. Secondly, if the order is of sufficiently small value, then no legal assistance should be sought. If both these policies were applied to the workflow, then it would essentially be represented as ReceiveOrder $\rightarrow$ IdentifyOffer $\rightarrow$ Create Contract.

All tasks inside the workflow are handled by services, either internal to the supplier or external (e.g. in the case of legal assistance and getting customer acceptance or refusal). The service composition and configuration can then be modelled formally in SRML. The effect of the policies can also be incorporated and through the application to the SRML model, the effects can be analysed and reasoned about.

Any method suggested for software engineering must be considered with respect to its scalability. The term can be interpreted in two different ways here, namely does the encoding mechanism itself scale and also do the STPOWLA and SRML methods scale. To answer the former, it can be said that it scales as every opeartor of STPOWLA is mapped into a relatively straight forward transition in SRML. The only exception is the random choice operator, where the matching transition is slightly more complex. As for the latter aspect, and in our opinion this is more crucial for practical purposes, we 
actually gain scalability. This is achieved by using the high-level STPOWLA notation to capture the business process in a more abstract and more easily understandable way than one would achieve by using SRML directly. However reasoning for e.g. validation can make use of the formality of SRML in addition of course to SRML being a step towards implementation.

\section{Summary and Conclusion}

The engineering of Service Oriented applications, as opposed to more traditional application development, is faced with novel challenges arising from the dynamicity of component selection and assembly, leading to massively distributed, interoperable and evolvable systems. Furthermore, a continuing challenge is to correctly align business goals with IT strategy. As such, the development approach must change to accommodate these factors.

In this paper, we have presented a mapping from STPOWLA to SRML. SRML is a high level modelling language for service-based applications, based on a formal framework. SRML can model service compositions and configurations. The orchestration of the services is modelled by a central agent in each SRML module. However, the business process aspect is less clear. STPOWLA is an SOA aware approach that combines workflows and policies. It allows to define the orchestration according to a business process.

The main contributions of this report are 1) to encode basic STPOWLA workflow constructs in SRML, 2) to extend STPOWLA with workflow reconfiguration functions, and 3) to encode these reconfiguration functions in SRML.

Future work includes the application of this work to some larger case studies and consider mapping STPOWLA refinement policies into SRML.

\section{References}

1. Abreu, J., Bocchi, L., Fiadeiro, J.L., Lopes, A.: Specifying and Composing Interaction Protocols for Service-Oriented System Modelling. In: Derrick, J., Vain, J. (eds.) FORTE 2007. LNCS, vol. 4574, pp. 358-373. Springer, Heidelberg (2007)

2. Adams, M., ter Hofstede, A.H.M., Edmond, D., van der Aalst, W.M.P.: Worklets: A serviceoriented implementation of dynamic flexibility in workflows. In: Meersman, R., Tari, Z. (eds.) OTM 2006. LNCS, vol. 4275, pp. 291-308. Springer, Heidelberg (2006)

3. Bocchi, L., Hong, Y., Lopes, A., Fiadeiro, J.L.: From BPEL to SRML: A Formal Transformational Approach. In: Proc.of 4th International Workshop on Web Services and Formal Methods (WSFM 2007). LNCS, Springer, Heidelberg (2007)

4. Dustdar, S., Fiadeiro, J.L., Sheth, A.P. (eds.): BPM 2006. LNCS, vol. 4102. Springer, Heidelberg (2006)

5. Fiadeiro, J.L., Lopes, A., Bocchi, L.: A Formal Approach to Service Component Architecture. Web Services and Formal Methods 4184, 193-213 (2006)

6. Gorton, S., Montangero, C., Reiff-Marganiec, S., Semini, L.: StPowla: SOA, Policies and Workflows. In: Proceedings of 3rd International Workshop on Engineering Service-Oriented Applications: Analysis, Design, and Composition, Vienna, Austria, September 17, 2007 (2007) 
7. Gorton, S., Reiff-Marganiec, S.: Towards a task-oriented, policy-driven business requirements specification for web services. In: Dustdar, et al. (eds.) [4], pp. 465-470

8. Haas, H., Brown, A.: Web Services Glossary. W3C Working Group Note, World Wide Web Consortium (W3C) (2004), http://www.w3.org/TR/ws-gloss/

9. Beisiegel, M., Blohm, H., Booz, D., Dubray, J., Colyer, A., Edwards, M., Ferguson, D., Flood, B., Greenberg, M., Kearns, D., Marino, J., Mischkinsky, J., Nally, M., Pavlik, G., Rowley, M., Tam, K., Trieloff, C.: Building Systems using a Service Oriented Architecture. Whitepaper, SCA Consortium (2005), http://www.oracle.com/technology/ tech/webservices/standards/sca/pdf/SCA_Nhite_Paper1_09.pdf

10. Montangero, C., Reiff-Marganiec, S., Semini, L.: Logic-Based Detection of Conflicts in Appel Policies. In: Arbab, F., Sirjani, M. (eds.) FSEN 2007. LNCS, vol. 4767, pp. 257-271. Springer, Heidelberg (2007)

11. Moses, T.: extensible access control markup language specification (2005), www.oasisopen.org

12. Müller, R., Greiner, U., Rahm, E.: Agentwork: a workflow system supporting rule-based workflow adaptation. Data Knowl. Eng. 51(2), 223-256 (2004)

13. Reiff-Marganiec, S.: Policies: Giving users control over calls. In: Ryan, M.D., Meyer, J.J.C., Ehrich, H.-D. (eds.) Objects, Agents, and Features, Dagstuhl Seminar 2003. LNCS, vol. 2975, pp. 189-208. Springer, Heidelberg (2004)

14. Reiff-Marganiec, S., Turner, K.J., Blair, L.: APPEL: the ACCENT project policy environment/language. Technical Report TR-161, University of Stirling (2005)

15. Russell, N., van der Aalst, W.M.P., ter Hofstede, A.H.M., Wohed, P.: On the suitability of uml 2.0 activity diagrams for business process modelling. In: Stumptner, M., Hartmann, S., Kiyoki, Y. (eds.) APCCM. CRPIT, vol. 53, pp. 95-104. Australian Computer Society (2006)

16. van der Aalst, W.M.P., ter Hofstede, A.H.M.: Yawl: yet another workflow language. Inf. Syst. 30(4), 245-275 (2005)

17. van der Aalst, W.M.P., ter Hofstede, A.H.M., Kiepuszewski, B., Barros, A.P.: Workflow patterns. Distributed and Parallel Databases 14(1), 5-51 (2003), Information, www.workflowpatterns.com

18. Wang, H.J.: A Logic-based Methodology for Busines Process Analysis and Design: Linking Business Policies to Workflow Models. PhD thesis, University of Arizona (2006)

19. White, S.A.: Business process modelling notation. Object Management Group (OMG) and Business Process Management Initiative (2004), www.bpmn.org

20. Wirsing, M., Bocchi, L., Clark, A., Fiadeiro, J.L., Gilmore, S., Hölzl, M., Koch, N., Pugliese, R.: SENSORIA: Engineering for Service-Oriented Overlay Computers, June 2007. MIT, Cambridge (submited 2007)

21. Wohed, P., Aalst, W.M.P.v.d., Dumas, M., Hofstede, A.H.M.t., Russell, N.: On the suitability of bpmn for business process modelling. In: Dustdar, et al. (eds.) [4], pp. 161-176 